\title{
Clinical outcome and pathologic correlation of stereotactic body radiation therapy as a bridge to transplantation for advanced hepatocellular carcinoma: a case series
}

\author{
Ying-Fu Wang ${ }^{1}$, Yang-Hong Dai ', Chun-Shu Lin ${ }^{1}$, Hao-Chih Chang ${ }^{1}$, Po-Chien Shen ${ }^{1}$, Jen-Fu Yang ${ }^{1}$, \\ Chih-Weim Hsiang ${ }^{2}$, Cheng-Hsiang Lo ${ }^{1^{*}}$ and Wen-Yen Huang ${ }^{1 *}$ (I)
}

\begin{abstract}
Background: Stereotactic body radiotherapy (SBRT) is an emerging modality for hepatocellular carcinoma (HCC). However, there is scant information about its safety and effectiveness in the neoadjuvant setting prior to liver transplantation (LT). We present the clinical outcome and pathologic assessment of SBRT followed by LT for patients with advanced HCC.

Methods: This retrospective study included HCC patients treated with neoadjuvant SBRT prior to LT between 2009 and 2018. Radiographic response and adverse effects, including radiation-induced liver disease (RILD), were evaluated. Pathologic response was assessed by the percentage of tumor necrosis relative to the total tumor volume. Overall survival (OS) and recurrence-free survival (RFS) were calculated using the Kaplan-Meier method.

Results: Fourteen patients underwent SBRT for a total of $25 \mathrm{HCC}$ lesions, followed by LT. The median tumor size was $4.45 \mathrm{~cm}$ in diameter, and the median prescribed dose was $45 \mathrm{~Gy}$ in 5 fractions. SBRT provided significant AFP reduction, $100 \%$ infield control, and a $62.5 \%$ response rate. The maximum detected toxicity included grade 3 thrombocytopenia and two grade 3-4 hyperbilirubinemia. One patient developed non-classic RILD. Patients were bridged to LT with a median time of 8.4 months after SBRT, and $23.1 \%$ of them achieved a complete pathologic response. The median OS and RFS were 37.8 and 18.3 months from the time of LT, respectively.
\end{abstract}

Conclusions: SBRT provides favorable tumor control and acceptable adverse effects for patients awaiting LT. Further prospective studies to test SBRT as a bridging therapy for LT are feasible.

Keywords: Hepatocellular carcinoma, Liver transplantation, Radiotherapy, Stereotactic body radiotherapy

\section{Background}

Hepatocellular carcinoma (HCC) is the most common primary hepatic malignancy and a leading cause of cancer mortality worldwide [1]. Most HCCs are caused by

\footnotetext{
*Correspondence: Isir183@yahoo.com.tw; hwyyi@yahoo.com.tw ${ }^{1}$ Department of Radiation Oncology, Tri-Service General Hospital, National Defense Medical Center, No. 325, Cheng-Gong Rd, Neihu, Taipei 114, Taiwan

Full list of author information is available at the end of the article
}

chronic liver disease; for instance, HCCs among Asians are caused by chronic viral hepatitis. Liver transplantation (LT) is a potentially curative therapy for HCC and is the best option for patients with cirrhosis and portal hypertension. The Milan criteria and the expanded set of criteria proposed by the University of California San Francisco (UCSF criteria) are well-validated guidelines for LT $[2,3]$. Through tumorous liver removal and liver function correction, LT provides excellent local control 
and leads to a 4-year post-transplant survival rate of $85 \%$ and recurrence-free survival rate of $92 \%[2,4]$.

Unfortunately, only a small percentage of HCC patients could receive transplants due to the scarcity of donors and the great number of nonmalignant indications. Furthermore, HCC patients are at risk of tumor progression, making them ineligible for transplantation that involves a long waiting period, which further results in a higher dropout rate compared to nonmalignant candidates (31.8\% vs. $19.1 \%$ at 1 year) after listing for LT [5]. Various locoregional therapies serve as a bridging strategy that aim to prevent the dropping out of waitlisted patients, or as a downstaging strategy to convert advanced HCC to LT candidates. Transarterial chemoembolization (TACE) and radiofrequency ablation (RFA) have been the most commonly used approaches in controlling tumor growth and vascular invasion, with a reportedly low dropout rate of $12.2 \%$ [6].

Historically, hepatic radiotherapy was limited due to the low radiation tolerance of the liver and the potential for radiation-induced liver disease (RILD). With precise delivery of an ablative dose of radiation to the target tumor within a limited number of fractions, stereotactic body radiotherapy (SBRT) has been developed as a safe and effective locoregional therapy for both primary and metastatic hepatic neoplasms [7]. SBRT was reported to achieve a 2-year local control rate of $74-100 \%$ and overall survival rate of $34-68.7 \%$ that are comparable with outcomes after RFA and TACE [8]. However, the effectiveness and safety of SBRT in the neoadjuvant setting are still under investigation [9-17], and information on clinical outcomes and pathological responses after irradiation are still scarce. Here, we report the clinical outcomes of neoadjuvant SBRT followed by LT for patients with advanced HCC, as well as the pathologic evaluation of HCC lesions treated with radiotherapy.

\section{Methods}

We retrospectively reviewed the medical records of $\mathrm{HCC}$ patients treated with neoadjuvant SBRT in our institution from January 2009 to December 2018. The inclusion criteria were as follows: (1) patients with confined HCC without extrahepatic metastases, (2) an Eastern Cooperative Oncology Group performance status of $\leq 2$, and (3) no previous abdominal radiotherapy. Pathologic diagnosis of HCC was not required as long as established radiographic criteria were satisfied for diagnosis [18]. SBRT was used in bridging or downstaging prior to LT. Other prior liver-directed therapies for HCC have been allowed. Our institutional review board (IRB) approved this study and waived the requirement for informed consent owing to the retrospective nature of the study.

\section{SBRT planning and treatment}

All patients were immobilized using a vacuum cushion in the supine position during simulation and treatment. Contrast enhanced computed tomography (CT) simulation with $3 \mathrm{~mm}$ slice thickness was performed, and previous dynamic CT or magnetic resonance imaging (MRI) were used as a reference to determine disease extent.

For patients with multiple tumors and preserved liver function, we would like to treat all lesions with SBRT as possible with respect to the dose constraints of the critical organs, especially the normal liver. If the SBRT plan could not cover all lesions, we would like to target the portal vein tumor thrombosis (PVTT) and the index tumor. The gross tumor volume (GTV) was defined as an enhancing tumor seen on CT and/or MRI. The planning target volume (PTV) was created by expanding the 0 - to 8-mm margin around the GTV. Modification of the PTV was acceptable when overlapping the dose-limiting organs, except for the normal liver. The radiation dose was prescribed to PTV individually based on the normal organ dose constraints determined by the institutional protocol.

SBRT was delivered using either the CyberKnife system (Accuray Inc., Sunnyvale, CA) or TomoTherapy system (Accuray Inc., Sunnyvale, CA). Patients treated using CyberKnife were treated with respiratory tracking of the tumor via peritumoral fiducials. For patients treated using TomoTherapy, breathing motion management was conducted with abdominal compression to reduce liver motion and with four-dimensional CT images to estimate internal target volume.

\section{Response and toxicity evaluation}

All patients were assessed during the entire course of SBRT, at 1- to 3-month intervals after completion of SBRT until orthotopic LT, at 3- to 4-month intervals for the first 2 years after surgery, and at 6-month intervals thereafter. Clinical evaluation, complete blood count, liver function, Child-Pugh score, alpha-fetoprotein (AFP), toxicity, and imaging with either contrast-enhanced dynamic CT or MRI were performed. Radiographic response to SBRT was assessed according to the modified Radiographic Evaluation Criteria in Solid Tumors (mRECIST) [19]. In-field failure was defined as disease progression or new enhancement within the PTV or at its margins. For patients with AFP elevation $(\geq 20 \mathrm{IU} / \mathrm{mL})$ before SBRT, reduction in AFP was calculated using the pretreatment baseline minus the minimal value after irradiation, and was censored at the time of intrahepatic progression, liver-directed therapies, or transplant. Acute and late adverse effects were graded using the National Cancer Institute Common 
Terminology Criteria for Adverse Events v4.0. RILD was established in the absence of intrahepatic disease progression (based on mRECIST criteria) within 3 months after completion of SBRT, and was recorded in two distinct entities. Classic RILD includes anicteric hepatomegaly, nonmalignant ascites, and elevated alkaline phosphatase of at least twice the upper normal limit. The non-classic RILD is composed of liver transaminase elevation of more than 5 times the reference value, or worsening of liver metabolic function represented as an increase of 2 or more points in the Child-Pugh score. For patients with preexisting abnormal transaminase and/ or alkaline phosphatase, the pretreatment baseline was adopted rather than the upper limits of the normal range. Patients were reassessed for transplant candidates using the Milan criteria, and underwent either deceased donor liver transplantation (DDLT) or living donor liver transplantation (LDLT), as clinically appropriate.

After the LT, we evaluated the SBRT treatment effect through pathological assessment. The pathologic response was estimated as the percentage of tumor necrosis relative to the total tumor volume, as follows: complete pathologic response was $100 \%$ tumor necrosis and the absence of any viable tumor; significant pathologic response was 50-99\% tumor necrosis in crosssection; minimal pathologic response was $1-49 \%$ tumor necrosis; and no pathologic response was no tumor necrosis present [11]. The correlation between pathologi$\mathrm{cal}$ response and radiologic response based on the mRECIST criteria was analyzed using the Pearson correlation coefficient. Disease recurrence, actuarial overall survival (OS), and recurrence-free survival (RFS) were analyzed from the time of transplantation using the Kaplan-Meier method.

\section{Results \\ Patients}

We retrospectively screened 188 patients who received SBRT at our institution between 2009 and 2018. Fourteen patients were included in this study (Fig. 1), and the baseline characteristics at the time of SBRT are listed in Table 1. The median age was 55.5 years, and most patients had underlying hepatitis B virus infection (78.6\%) and preserved liver function (Child-Pugh class A, 78.6\%). Thirteen patients have received prior liverdirected treatment for $\mathrm{HCC}$ in the form of TACE alone

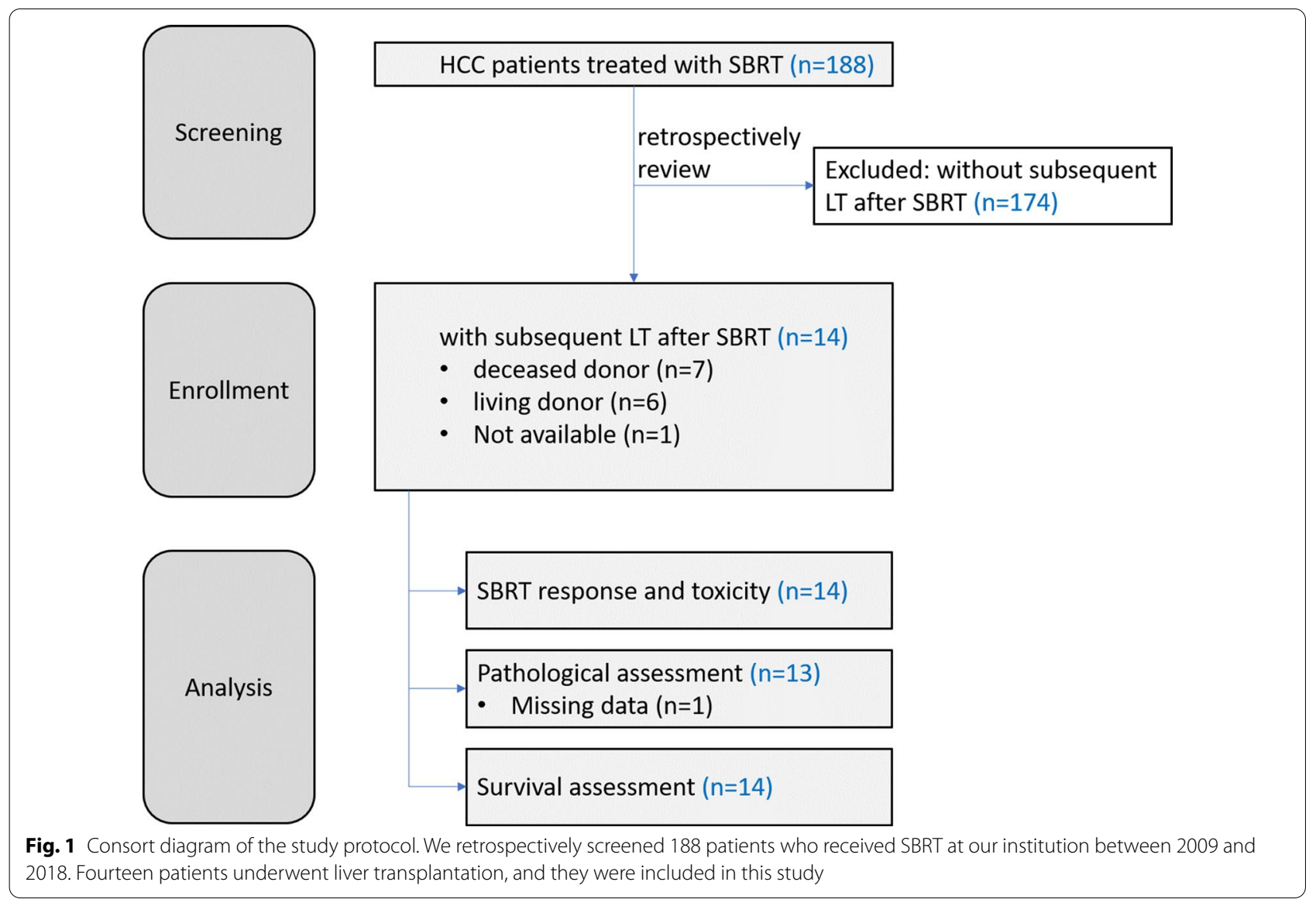


Table 1 Patient characteristics at the first time of SBRT $(n=14)$

\begin{tabular}{|c|c|}
\hline Characteristics & \\
\hline Age, median (range) & $55.5(40-68)$ \\
\hline Male, no. (\%) & $12(85.7)$ \\
\hline \multicolumn{2}{|l|}{ Cause of cirrhosis, no. (\%) } \\
\hline HBV & $11(78.6)$ \\
\hline $\mathrm{HCV}$ & $2(14.3)$ \\
\hline Alcoholic & $1(7.1)$ \\
\hline \multicolumn{2}{|l|}{ ECOG performance status, no. (\%) } \\
\hline 0 & $9(64.3)$ \\
\hline 1 & $4(28.6)$ \\
\hline 2 & $1(7.1)$ \\
\hline \multicolumn{2}{|l|}{ Child-Pugh class, no. (\%) } \\
\hline A & $11(78.6)$ \\
\hline B & $2(14.3)$ \\
\hline C & $1(7.1)$ \\
\hline \multicolumn{2}{|l|}{ Previous treatment, no. (\%) } \\
\hline TACE & $7(50.0)$ \\
\hline RFA + TACE & $1(7.1)$ \\
\hline TACE + Thaldo & $2(14.3)$ \\
\hline Segmentectomy + TACE & $2(14.3)$ \\
\hline Segmentectomy + TACE + RFA & $1(7.1)$ \\
\hline None & $1(7.1)$ \\
\hline Max. tumor diameter, median (range) & $4.45(0.9-10.5)$ \\
\hline \multicolumn{2}{|l|}{ Number of tumors, no. (\%) } \\
\hline Solitary & $4(28.6)$ \\
\hline Multiple & $10(71.4)$ \\
\hline Portal venous tumor thrombosis, no. (\%) & $4(28.6)$ \\
\hline AFP, median (range), IU/mL & $59.5(3.0-1286.0)$ \\
\hline \multicolumn{2}{|l|}{ BCLC stage at SBRT, no. (\%) } \\
\hline A & $3(21.4)$ \\
\hline B & $6(42.9)$ \\
\hline $\mathrm{C}$ & $4(28.6)$ \\
\hline $\mathrm{D}$ & $1(7.1)$ \\
\hline Within Milan criteria before SBRT, no. (\%) & $4(28.6)$ \\
\hline
\end{tabular}

AFP, alpha-fetoprotein; BCLC, Barcelona clinic liver cancer; RFA, radiofrequency ablation; SBRT, stereotactic body radiation therapy; TACE, transcatheter arterial chemoembolization

or TACE combined with other modalities. Ten (71\%) patients had multifocal disease and 4 (28.7\%) was found to have PVTT. Four (28.6\%) patients met the Milan criteria for LT at the time of SBRT.

Sixteen SBRT courses with either CyberKnife $(n=15)$ or TomoTherapy system $(\mathrm{n}=1)$ have been conducted for a total of 25 HCC lesions. Two patients underwent repeated SBRT for intrahepatic recurrence between the first SBRT and LT. The SBRT treatment parameters and outcomes are shown in Table 2. The median prescribed dose was 45 Gy (range: 28-60 Gy) in 5 fractions (range:
4-5). The median volumes of treated tumors and the normal liver were $35.9 \mathrm{~mL}$ and $1281.7 \mathrm{~mL}$, respectively. Patient no. 7, a 55-year-old man, received a second SBRT due to intrahepatic out-field recurrence 12 months after the first SBRT. He underwent LDLT 11 months after the completion of the second SBRT. Patient no. 10 was a 58-year-old woman who experienced repeated intrahepatic recurrence, and she had been treated with wedge resection, 4 courses of TACE, 2 courses of SBRT (with 32 months interval), and 2 courses of RFA without major adverse events. Eventually, she underwent DDLT 40 months after finishing the second SBRT (8 years after HCC diagnosis).

There were 10 patients with treatment failure before LT, all in the form of intra-hepatic out-field failure. For out-field recurrence after SBRT, 8 patients received further salvage treatments (median, 1; range, 0-6 times) prior to LT, and 2 patients underwent LT without additional anti-HCC treatment. The first salvage treatment included SBRT $(n=1)$, TACE $(n=3)$, RFA $(n=1)$, multikinase inhibitor $(n=1)$, and TACE combined with a multi-kinase inhibitor $(n=2)$. Repeated intrahepatic out-field recurrence was noted after salvage treatment in 4 patients, and combined treatment modalities were arranged before LT.

\section{Response to SBRT as a bridge therapy}

Complete radiologic response was observed in $4(25 \%)$ of the 16 SBRT courses, partial response in 6 (37.5\%), and stable disease in 6 (37.5\%), with an infield control rate of $100 \%$ reached prior to LT (Table 2). For the 7 patients with pretreatment elevated AFP (median: $148.0 \mathrm{IU} /$ $\mathrm{mL}$ ), maximal AFP response (median: $38.3 \mathrm{IU} / \mathrm{mL}$ ) was observed at 1.5 months (range: $0.8-5.1$ months) after the first day of SBRT. After completion of SBRT, 1 of the 4 patients within the Milan criteria initially had intrahepatic out-field tumor progression and was dropped out. Among the 10 patients initially beyond the Milan criteria, 2 had tumors that shrunk and met transplant eligibility after SBRT.

All 14 patients completed the prescribed courses of SBRT. The most common adverse effects were grade 1-2 gastrointestinal toxicities. Three (18.8\%) patients developed grade 3 thrombocytopenia, and 2 (12.5\%) patients had grade 3 or 4 blood hyperbilirubinemia (Table 2). Non-classic RILD developed in 1 patient (patient no.8), who had alcoholic cirrhosis and HCC after TACE, with common hepatic duct invasion and PVTT. He received SBRT to the residual tumor at 35 Gy in 5 fractions, and the volume of the uninvolved liver that received $\leq 15$ Gy was $654 \mathrm{~mL}$. At 2 months after irradiation, acute suppurative cholangitis and non-classic RILD (Child-Pugh A6 to C12) developed. The hepatic toxicity recovered 
Table 2 SBRT parameters, outcomes, and adverse events $(n=16)$

\begin{tabular}{|c|c|c|c|c|}
\hline \multicolumn{5}{|c|}{ SBRT treatment, median (range) } \\
\hline \multicolumn{4}{|l|}{ SBRT dose, Gy } & $45.0(28-60)$ \\
\hline \multicolumn{4}{|l|}{ Number of fractions } & $5(4-5)$ \\
\hline \multicolumn{4}{|l|}{ EQD2, Gy } & $71.25(36.4-110.0)$ \\
\hline \multicolumn{4}{|l|}{ Tumor volume, cc } & $35.9(1.11-819.43)$ \\
\hline \multicolumn{4}{|l|}{ Number of targeted lesions } & $2(1-4)$ \\
\hline \multicolumn{4}{|l|}{ Normal liver volume, cc } & $1281.7(537.0-2095.1$ \\
\hline \multicolumn{3}{|l|}{ rV15* of uninvolved liver, cc } & & $1019.6(436.6-1610.2$ \\
\hline \multicolumn{5}{|l|}{ SBRT outcomes, no. (\%) } \\
\hline \multicolumn{5}{|l|}{ Radiographic response } \\
\hline \multicolumn{3}{|l|}{ Complete response (rCR) } & & $4(25)$ \\
\hline \multicolumn{3}{|l|}{ Partial response (rPR) } & & $6(37.5)$ \\
\hline \multicolumn{3}{|l|}{ Stable disease (rSD) } & & $6(37.5)$ \\
\hline \multicolumn{5}{|l|}{ Local recurrence } \\
\hline \multicolumn{3}{|l|}{ Out-field } & & $11(68.8)$ \\
\hline \multicolumn{3}{|l|}{ In-field } & & 0 \\
\hline AFP after SBRT**, median & & & & $38.3(2.8-386.3)$ \\
\hline Within Millan criteria after & & & & 5 of 14 patients (35.7) \\
\hline Adverse events, no. (\%) & Grade 1 & Grade 2 & Grade3 & Grade4 \\
\hline Biochemical & & & & \\
\hline Albumin & $3(18.8)$ & $3(18.8)$ & 0 & 0 \\
\hline Alkaline phosphatase & $1(6.3)$ & 0 & 0 & 0 \\
\hline ALT & $7(43.8)$ & 0 & 0 & 0 \\
\hline AST & $7(43.8)$ & 0 & 0 & 0 \\
\hline Bilirubin & 0 & $1(6.3)$ & $1(6.3)$ & $1(6.3)$ \\
\hline Hematologic & & & & \\
\hline Anemia & $3(18.8)$ & $1(6.3)$ & $1(6.3)$ & 0 \\
\hline Leukocytopenia & $6(37.5)$ & $1(6.3)$ & $1(6.3)$ & 0 \\
\hline Thrombocytopenia & $5(31.3)$ & $1(6.3)$ & $3(18.8)$ & 0 \\
\hline Fatigue & $2(12.5)$ & 0 & 0 & 0 \\
\hline Gastrointestinal toxicity & & & & \\
\hline Abdominal pain & $2(12.5)$ & 0 & 0 & 0 \\
\hline Anorexia & $1(6.3)$ & 0 & 0 & 0 \\
\hline Diarrhea & $1(6.3)$ & $1(6.3)$ & 0 & 0 \\
\hline Nausea/vomiting & $1(6.3)$ & $2(12.5)$ & 0 & 0 \\
\hline RILD, no. (\%) & & & & \\
\hline Classic & & & & $0(0)$ \\
\hline Nonclassic & & & & $1(6.3)$ \\
\hline
\end{tabular}

AST, aspartate aminotransferase; ALT, alanine aminotransferase; rCR, radiological complete response; rPR, radiological partial response; rSD, radiological stable disease; RILD, radiation induced liver disease; SBRT, stereotactic body radiation therapy

${ }^{*}$ rV15 was defined as volume of uninvolved liver receiving $\leq 15 \mathrm{~Gy}$ (cc)

**Only apply for patients with elevated AFP level at baseline

partially to Child-Pugh C10 after his cholangitis was controlled, and he underwent DDLT 8.6 months after SBRT.

Subsequently, all patients successfully underwent LT from deceased donors $(n=7)$ or living donors $(n=6)$.
Patient no. 13 underwent LT in mainland China, with the medical records of the donor type as well as the pathology report both unavailable. The median duration from the time of last SBRT to LT was 8.4 months 
(range: 1.6-62.4 months). There were no major perioperative complications.

Details regarding the 13 patients with evaluable surgical pathology are listed in Table 3. Twelve patients had residual HCC, of which 11 had multifocal tumors. Three patients had complete pathologic response, 3 had significant pathologic response, and 5 had minimal pathologic response. Information about tumor necrosis were insufficient in 2 patients. Poor concordance was observed, with a Pearson correlation coefficient of -0.434 between the percentage of necrosis and the post-SBRT radiologic response according to the mRECIST criteria.

\section{Outcome after LT}

The data were collected through June 2020 and the median follow-up from the time of transplantation was 30.2 months (range: $3.2-122.2$ months). Five patients were alive during the last follow-up. The median OS was 37.8 months (95\% CI: 0-91.96 months), and the median RFS was 18.3 months (95\% CI: 0-36.91 months) (Fig. 2). During the follow-up period after transplantation, five patients experienced recurrence (1 with hepatic recurrence and 4 with distant metastasis), and 4 of them died from the disease. Patient no. 13 developed solitary pulmonary metastasis 28 months after LT, and he survived with no evidence of disease after video-assisted thoracoscopic surgery with wedge resection.

The prognostic factors for recurrence-free survival and overall survival were analyzed. However, none of the factors had a significant survival impact in univariate analysis (Additional file 1: Table S1).

\section{Discussion}

This single institutional retrospective study showed that SBRT is an effective and safe modality in neoadjuvant settings even in advanced HCC. After SBRT, patients smoothly underwent LT with favorable pathological responses.

Several liver-directed therapies have been used as a neoadjuvant treatment to prevent HCC patients from dropping out of the transplant waiting list. Fisher et al. reported that aggressive multi-modality therapy with TACE and RFA might optimize the use of LT; this approach had a low dropout rate of $12.2 \%$ during a median waiting time of 9.1 months [6]. However, each modality is limited by the tumor size, number, and location, the adjacent critical organs, the liver function, and the patient's general condition. SBRT has been developed as an effective noninvasive bridge treatment with excellent tumor control and tolerable side effects (Table 4) in several observational studies [9-16], with doses of 30-54 Gy in 3-6 fractions. SBRT provides a low dropout rate and achieves a complete necrosis rate of $27-61.5 \%$ of the treated lesions, resulting in favorable overall and disease-free survival after LT.

Sapisochin et al. [17] from the University of Toronto reported an intention-to-treat analysis comparing SBRT with TACE and RFA as a bridge to LT in a large cohort of 406 HCC patients. The calculated model for endstage liver disease score was slightly higher for the SBRT group, and only $36 \%$ of them were within the Milan criteria preoperatively, whereas $23 \%$ in the TACE group and $87.7 \%$ in the RFA group were within the Milan criteria. The median prescribed radiation dose was 36 Gy (interquartile range: $30-40$ Gy) in 6 fractions. SBRT provided a $16.7 \%$ dropout rate from the waiting list, similar to those of patients treated with TACE $(20.2 \%)$ or RFA (16.8\%). Although the 5-year cumulative risk of posttransplant recurrence was better in the RFA group, there was no difference in the 5-year posttransplant survival among the three modalities (75\% in SBRT, 69\% in TACE, and 73\% in RFA group). These comparative data suggested that SBRT can be safely utilized as a bridge to LT in patients with HCC. It may offer advantages when TACE or RFA are not applicable or fail to control the tumor.

By contrast, our cohort was in a relatively advanced stage, with only $28.6 \%$ patients initially meeting the Milan criteria. The selection of LT candidates relies mostly on tumor size and number. In principle, Milan criteria were utilised for HCC recipients with deceased donor grafts, and expanded criteria of UCSF were used for living donor LT. For patients with lesions exceeding the Milan criteria, tumor differentiation and cancer-related symptoms were also considered regardless of the tumor size and number, as the so-called extended Toronto criteria [20]. During our study period, the size and number of tumors were not absolute contraindications to LT. However, patients with extrahepatic disease were absolutely excluded from the waiting list. Given that AFP levels $>500 \mathrm{ng} / \mathrm{mL}$ were predictive of poor survival outcomes for patients within or beyond the Milan criteria, AFP level was also incorporated for decision-making purposes [20]. In present study, SBRT still provided significant AFP reduction, $100 \%$ infield control, and $62.5 \%$ response rate, which were similar to the published series $[9,11-14,16]$. Five patients either underwent downstaging or were kept from dropout, and all 14 patients successfully underwent LT with a median duration from SBRT to LT of 8.4 months, which was longer than that reported in other series [9, 11-14, 16, 17]. However, the actuarial dropout rate was unevaluable in this study because we did not enroll all the patients who received SBRT with bridging intent, but only the patients who received SBRT and LT.

In our cohort, 4 (28.6\%) patients had PVTT at the time of SBRT. Their PVTT was included in the SBRT target. According to the BCLC guidelines, patients with $\mathrm{HCC}$ 


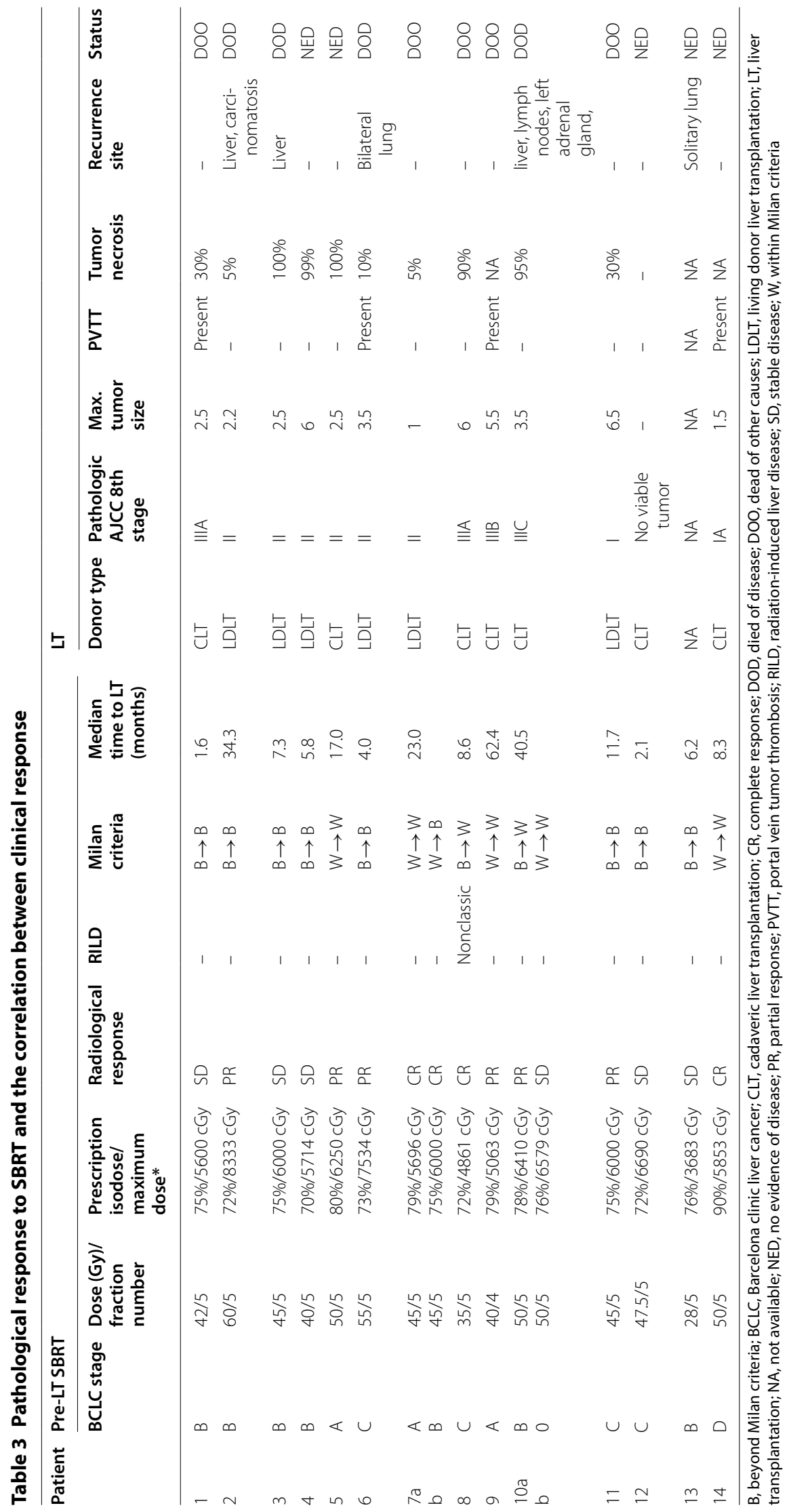




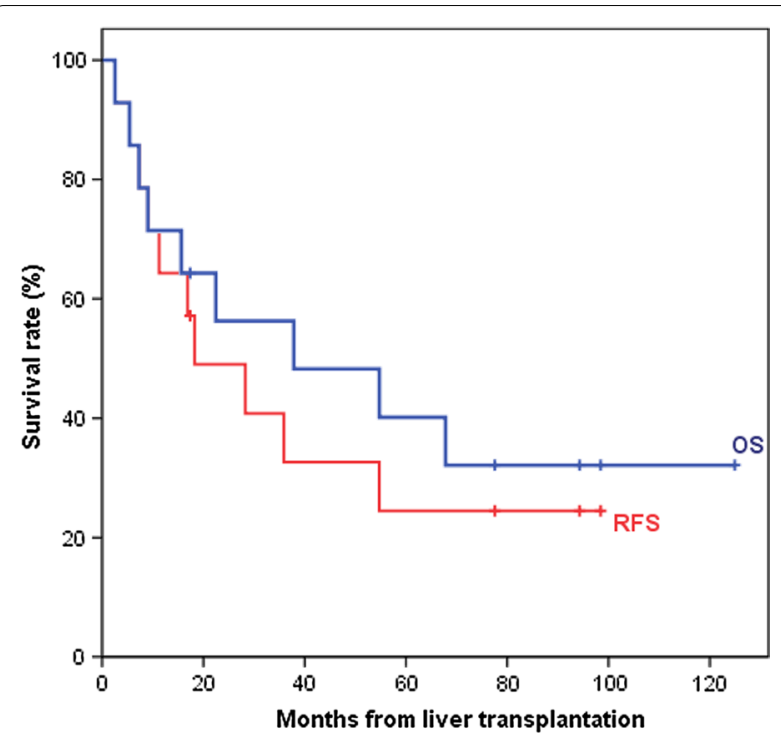

Fig. 2 Kaplan-Meier survival curves for overall survival (OS) and recurrence-free survival (RFS) in patients with HCC treated with neoadjuvant SBRT and LT. The median OS and RFS were 37.8 and 18.3 months, respectively

and PVTT are usually administered systemic therapy with/without palliative locoregional therapies. However, the presence of PVTT is no longer considered an absolute contraindication to LT; LT remains a critical part of the treatment algorithm [21-23]. In our institute, SBRT is part of the multimodality approach; properly selected patients will be treated with SBRT to the PVTT and index lesion(s). After SBRT, LT could be reconsidered individually at a multidisciplinary board for patients who respond to their $\mathrm{Vp} 3 / 4$ or stable disease during the waiting period.

There is considerable variation among the pathologic complete response rates reported in previous studies. Guarneri et al. achieved a $61.5 \%$ pathologic complete response after neoadjuvant SBRT [13], whereas in a study by Sapisochin et al., it was only 13.3\% [17]. In our cohort, three of the 13 (23.1\%) evaluable patients achieved complete tumor necrosis upon pathological analysis. All irradiated lesions had partial or complete pathologic response with shrinkage or necrosis of the tumor.

In contrast to the reportedly good concordance between pre-transplant radiologic response (according to the mRECIST criteria) and degree of necrosis in patients receiving neoadjuvant TACE [24], no strong correlation between radiologic and pathologic response after SBRT was observed in our study. Mannina et al. assessed the correlation between surgical pathology and radiologic scoring criteria in 38 patients treated with SBRT prior to LT [15]. They demonstrated the poor concordance of pathologic response with mRECIST (sensitivity, 90\%; specificity, 17\%), RECIST (sensitivity 54\%, specificity 50\%), and European Association for the Study of the Liver (EASL) criteria (sensitivity $83 \%$, specificity $18 \%$ ). Unlike the immediate post-treatment decreased- or non-enhancement following RFA and TACE, persistent arterial phase hyperenhancement for at least 12 months is common post SBRT and does not necessarily indicate viable neoplasm [25]. In addition to the mRECIST criteria, the AFP level as well as the apparent diffusion coefficient calculated from MRI sequences could improve the assessment of SBRT response and help to determine the LT candidates [26, 27].

The role of radiation therapy in the management of HCC remains to be limited because of concerns about liver toxicity and RILD. Some cases have been reported to have RILD after SBRT prior to LT. Guarneri et al. reported that one patient developed non-classic RILD 1 month after SBRT with 48 Gy in 3 fractions, and he underwent LT 2.2 months later [13]. Moore et al. reported a patient with Child-Pugh B8 who manifested with RILD after SBRT with $30 \mathrm{~Gy}$ in 5 fractions and underwent urgent LT due to hepatic decompensation [14]. The possible causative factors included underlying cirrhosis, low normal liver volume, and reirradiation. Nevertheless, irradiation had been well tolerated as a bridging therapy in our study, with minimal grade 3 or higher toxicity. One (6.3\%) patient in our cohort experienced non-classic RILD, which was consistent with the rate reported in previous studies $(0.0-12.5 \%)$. We did not observe significant surgical complications after SBRT in our cohort. However, the precise relationship between preoperative SBRT and transplant complications, such as rejection or biliary stricture, merits further study.

In our study, 1 patient experienced hepatic recurrence and 4 had distant metastases at the end of follow-up. The median OS was 37.8 months from the time of LT, which was worse than the data reported in previous studies [12, 15-17]. The relatively high recurrence rate and modest post-transplant survival in our cohort were probably due to the advanced disease, along with multinodular disease and/or PVTT. All patients were unsuitable or refractory to other locoregional therapies. In addition, the high recurrence rate could also be explained by the fact that many of the patients in our cohort remained beyond the Milan criteria even after SBRT and underwent LT with a living donor. Otto et al. reported that $88 \%$ of patients who successfully bridged or downstaged to fit the Milan criteria were recurrence-free in the 5 years post-LT, compared to $55 \%$ of those still exceeding the Milan criteria after neoadjuvant TACE [28]. Appropriate LT candidate selection after SBRT is therefore crucial. 


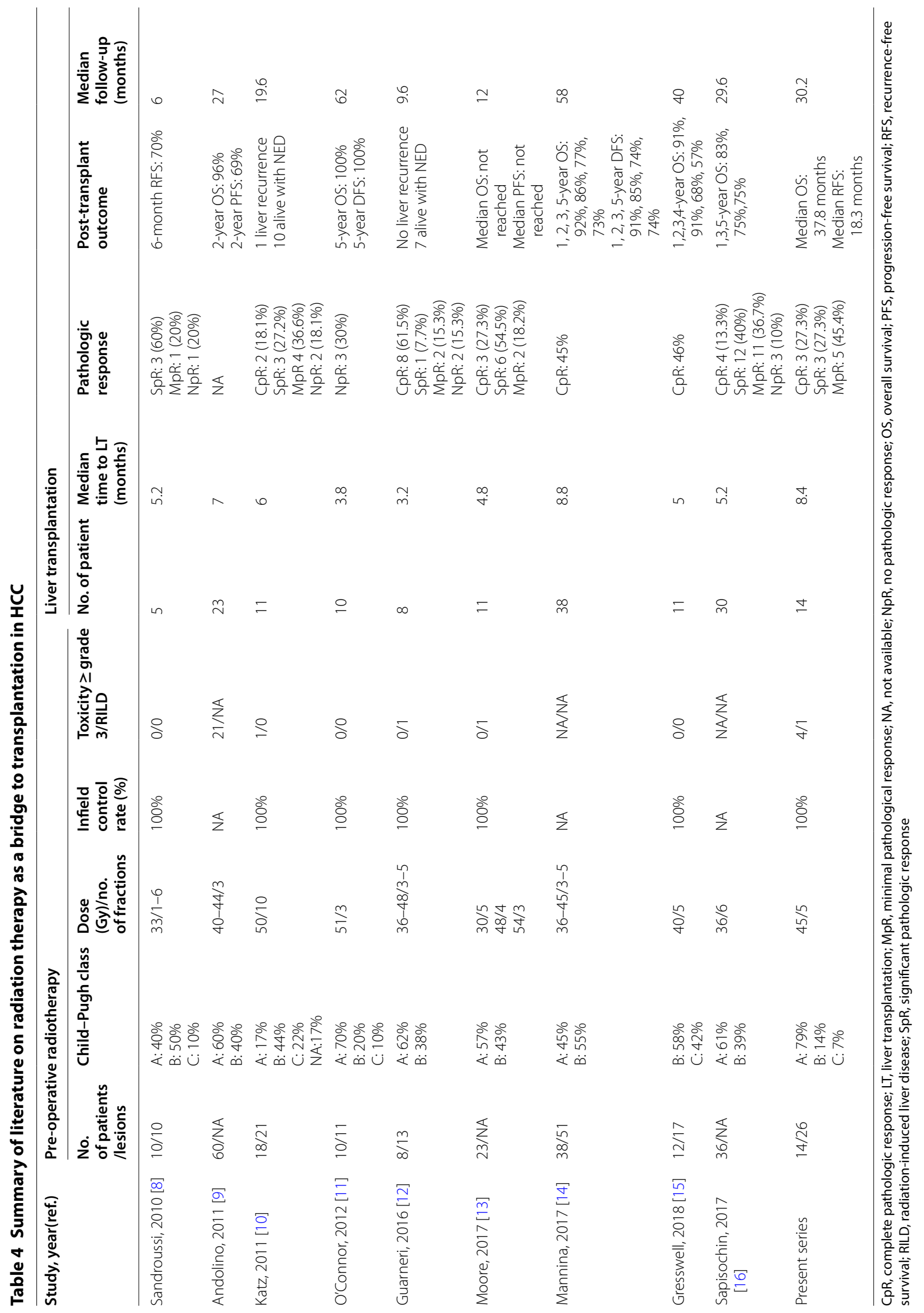


The main limitations of our study include its small case number and the heterogeneity of patient characteristics, prior treatments, and radiation dose. In addition, the retrospective study design and potential selection bias are also key limitations, and no conclusion can be drawn about the actuarial dropout rate and outcome compared to other therapies. However, there are limited reports on the pathologic evaluation post SBRT, and our experience contributes to the expansion of knowledge regarding this noninvasive treatment.

\section{Conclusions}

In conclusion, SBRT is safe and effective as a bridging or downstaging therapy for patients awaiting LT for HCC. SBRT provides favorable tumor control and minimal adverse effects and achieves good pathological response and posttransplant survival. These findings suggest that SBRT is a reasonable preoperative option for patients with advanced HCC.

\section{Supplementary information}

The online version contains supplementary material available at https://doi. org/10.1186/s13014-020-01739-5.

Additional file 1: Table S1: Prognostic factors influencing RFS and OS after LT using the Cox proportional hazards model.

\section{Abbreviations \\ AFP: Alpha-fetoprotein; CT: Computed tomography; DDLT: Deceased donor liver transplantation; GTV: Gross tumor volume; HCC: Hepatocellular carci- noma; LT: Liver transplantation; LDLT: Living donor liver transplantation; MRI: Magnetic resonance imaging; mRECIST: Modified Radiographic Evaluation Criteria in Solid Tumors; OS: Overall survival; PTV: Planning target volume; RILD: Radiation-induced liver disease; RFA: Radiofrequency ablation; RFS: Recurrence-free survival; SBRT: Stereotactic body radiotherapy; TACE: Tran- sarterial chemoembolization; UCSF criteria: Expanded set of criteria proposed by the University of California San Francisco.}

\section{Acknowledgement}

The authors thank the Cancer Registry Group of Tri-Service General Hospital for the clinical data support.

\section{Authors' contributions}

YFW contributed to study concepts, study design, literature research, definition of intellectual content, data acquisition, data analysis, data interpretation, statistical analysis, manuscript preparation and manuscript editing. YHD and CSL contributed to literature research, definition of intellectual content, data analysis, data interpretation, manuscript editing, and manuscript review. HCC, PCS, and JFY collected data, carried out the initial analyses, and reviewed and revised the manuscript. CHL and WYH conceptualized the study, coordinated, and supervised data collection, and provided critical editing and revision to the final drafts of the report. All authors made substantial intellectual contributions to drafting the article, revising the article, data analysis, and data interpretation. All authors have read and approved the final manuscript.

\section{Funding}

This study was supported by study project of Tri-Service General Hospital (TSGH-D-110086 \& TSGH-D-110087).

\section{Availability of data and materials}

All data generated or analyzed during this study are included in this published article.

\section{Ethics approval and consent to participate}

The protocol was established, according to the ethical guidelines of the Helsinki Declaration and was approved by the human ethics committee of Institutional Review Board of Tri-Service General Hospital. The requirement for informed consent was waived owing to the retrospective nature of the study.

\section{Consent for publication}

Not applicable.

\section{Competing interests}

The authors declare that they have no competing interests.

\section{Author details}

${ }^{1}$ Department of Radiation Oncology, Tri-Service General Hospital, National Defense Medical Center, No. 325, Cheng-Gong Rd, Neihu, Taipei 114, Taiwan. ${ }^{2}$ Department of Radiology, Tri-Service General Hospital, National Defense Medical Center, No. 325, Cheng-Gong Rd, Neihu, Taipei 114, Taiwan.

Received: 19 September 2020 Accepted: 26 December 2020 Published online: 14 January 2021

\section{References}

1. Villanueva A. Hepatocellular carcinoma. N Engl J Med. 2019;380(15):1450-62.

2. Mazzaferro V, Regalia E, Doci R, Andreola S, Pulvirenti A, Bozzetti F, et al. Liver transplantation for the treatment of small hepatocellular carcinomas in patients with cirrhosis. N Engl J Med. 1996;334(11):693-9.

3. Yao FY, Ferrell L, Bass NM, Watson JJ, Bacchetti P, Venook A, et al. Liver transplantation for hepatocellular carcinoma: expansion of the tumor size limits does not adversely impact survival. Hepatology. 2001;33(6):1394-403.

4. Mazzaferro V, Llovet JM, Miceli R, Bhoori S, Schiavo M, Mariani L, et al. Predicting survival after liver transplantation in patients with hepatocellular carcinoma beyond the Milan criteria: a retrospective, exploratory analysis. Lancet Oncol. 2009;10(1):35-43.

5. Freeman RB, Edwards EB, Harper AM. Waiting list removal rates among patients with chronic and malignant liver diseases. Am J Transplant. 2006;6(6):1416-21.

6. Fisher RA, Maluf D, Cotterell AH, Stravitz T, Wolfe L, Luketic V, et al. Nonresective ablation therapy for hepatocellular carcinoma: effectiveness measured by intention-to-treat and dropout from liver transplant waiting list. Clin Transplant. 2004;18(5):502-12.

7. Mazzola R, Fersino S, Alongi P, Di Paola G, Gregucci F, Aiello D, et al. Stereotactic body radiation therapy for liver oligometastases: predictive factors of local response by (18)F-FDG-PET/CT. Br J Radiol. 2018;91(1088):20180058.

8. Shen PC, Chang WC, Lo CH, Yang JF, Lee MS, Dai YH, et al. Comparison of stereotactic body radiation therapy and transarterial chemoembolization for unresectable medium-sized hepatocellular carcinoma. Int J Radiat Oncol Biol Phys. 2019;105(2):307-18.

9. Sandroussi C, Dawson LA, Lee M, Guindi M, Fischer S, Ghanekar A, et al. Radiotherapy as a bridge to liver transplantation for hepatocellular carcinoma. Transpl Int. 2010;23(3):299-306.

10. Andolino DL, Johnson CS, Maluccio M, Kwo P, Tector AJ, Zook J, et al. Stereotactic body radiotherapy for primary hepatocellular carcinoma. Int J Radiat Oncol Biol Phys. 2011;81(4):e447-53.

11. Katz AW, Chawla S, Qu Z, Kashyap R, Milano MT, Hezel AF. Stereotactic hypofractionated radiation therapy as a bridge to transplantation for hepatocellular carcinoma: clinical outcome and pathologic correlation. Int J Radiat Oncol Biol Phys. 2012;83(3):895-900.

12. O'Connor JK, Trotter J, Davis GL, Dempster J, Klintmalm GB, Goldstein RM. Long-term outcomes of stereotactic body radiation therapy in the treatment of hepatocellular cancer as a bridge to transplantation. Liver Transpl. 2012;18(8):949-54. 
13. Guarneri A, Franco P, Romagnoli R, Trino E, Mirabella S, Molinaro L, et al. Stereotactic ablative radiation therapy prior to liver transplantation in hepatocellular carcinoma. Radiol Med. 2016;121(11):873-81.

14. Moore A, Cohen-Naftaly M, Tobar A, Kundel Y, Benjaminov O, Braun M, et al. Stereotactic body radiation therapy (SBRT) for definitive treatment and as a bridge to liver transplantation in early stage inoperable Hepatocellular carcinoma. Radiat Oncol. 2017:12(1):163.

15. Mannina EM, Cardenes HR, Lasley FD, Goodman B, Zook J, Althouse S, et al. Role of stereotactic body radiation therapy before orthotopic liver transplantation: retrospective evaluation of pathologic response and outcomes. Int J Radiat Oncol Biol Phys. 2017;97(5):931-8.

16. Gresswell S, Tobillo R, Hasan S, Uemura T, Machado L, Thai N, et al. Stereotactic body radiotherapy used as a bridge to liver transplant in patients with hepatocellular carcinoma and Child-Pugh score $\geq 8$ cirrhosis. J Radiosurg SBRT. 2018;5(4):261-7.

17. Sapisochin G, Barry A, Doherty M, Fischer S, Goldaracena N, Rosales R, et al. Stereotactic body radiotherapy vs. TACE or RFA as a bridge to transplant in patients with hepatocellular carcinoma. An intention-to-treat analysis. J Hepatol. 2017;67(1):92-9.

18. European Association for the Study of the Liver. Electronic address EEE, European Association for the Study of the L. EASL Clinical Practice Guidelines: management of hepatocellular carcinoma. J Hepatol. 2018;69(1):182-236.

19. Lencioni R, Llovet JM. Modified RECIST (mRECIST) assessment for hepatocellular carcinoma. Semin Liver Dis. 2010;30(1):52-60.

20. Sapisochin G, Goldaracena N, Laurence JM, Dib M, Barbas A, Ghanekar $A$, et al. The extended Toronto criteria for liver transplantation in patients with hepatocellular carcinoma: a prospective validation study. Hepatology. 2016;64(6):2077-88.

21. Shui Y, Yu W, Ren X, Guo Y, Xu J, Ma T, et al. Stereotactic body radiotherapy based treatment for hepatocellular carcinoma with extensive portal vein tumor thrombosis. Radiat Oncol. 2018;13(1):188.
22. Jeong BK, Choi HS, Kang KM, Jeong H, Lee YH, Ha IB, et al. Stereotacticbody radiotherapy for portal vein tumor thrombosis in hepatocellular carcinoma patients. J Clin Oncol. 2018;36(4):442.

23. Abhishek A, Kataria T, Gupta D, Basu T, Bisht SS, Goyal S, et al. Portal vein tumor thrombus irradiation: paving the way for liver transplant. Int J Radiat Oncol Biol Phys. 2016;96(2):E164.

24. Zhang W, Xu AH, Wang W, Wu YH, Sun QL, Shu C. Radiological appearance of hepatocellular carcinoma predicts the response to trans-arterial chemoembolization in patients undergoing liver transplantation. BMC Cancer. 2019;19(1):1041.

25. Mendiratta-Lala M, Masch W, Shankar PR, Hartman HE, Davenport MS, Schipper MJ, et al. Magnetic resonance imaging evaluation of hepatocellular carcinoma treated with stereotactic body radiation therapy: long term imaging follow-up. Int J Radiat Oncol Biol Phys. 2019;103(1):169-79.

26. Mendiratta-Lala M, Gu E, Owen D, Cuneo KC, Bazzi L, Lawrence TS, et al. Imaging findings within the first 12 months of hepatocellular carcinoma treated with stereotactic body radiation therapy. Int J Radiat Oncol Biol Phys. 2018;102(4):1063-9.

27. Lo CH, Huang WY, Hsiang CW, Lee MS, Lin CS, Yang JF, et al. Prognostic significance of apparent diffusion coefficient in hepatocellular carcinoma patients treated with stereotactic ablative radiotherapy. Sci Rep. 2019;9(1):14157.

28. Otto G, Schuchmann M, Hoppe-Lotichius M, Heise M, Weinmann A, Hansen $T$, et al. How to decide about liver transplantation in patients with hepatocellular carcinoma: size and number of lesions or response to TACE? J Hepatol. 2013;59(2):279-84.

\section{Publisher's Note}

Springer Nature remains neutral with regard to jurisdictional claims in published maps and institutional affiliations.
Ready to submit your research? Choose BMC and benefit from:

- fast, convenient online submission

- thorough peer review by experienced researchers in your field

- rapid publication on acceptance

- support for research data, including large and complex data types

- gold Open Access which fosters wider collaboration and increased citations

- maximum visibility for your research: over $100 \mathrm{M}$ website views per year

At BMC, research is always in progress.

Learn more biomedcentral.com/submissions 